\title{
The Impact of tagSNPs in CXCL16 Gene on the Risk of Myocardial Infarction in a Chinese Han Population
}

\author{
Shun Xu, ${ }^{1,2,3}$ Jie Cheng, ${ }^{1,2,4}$ Meng-yun Cai, ${ }^{1,2,3}$ Li-li Liang, ${ }^{1,2,3}$ Jin-ming Cen, \\ Xi-li Yang, ${ }^{5}$ Can Chen, ${ }^{6}$ Xinguang Liu, ${ }^{1,2,3}$ and Xing-dong Xiong ${ }^{1,2,3}$ \\ ${ }^{1}$ Institute of Aging Research, Guangdong Medical University, Dongguan, China \\ ${ }^{2}$ Guangdong Provincial Key Laboratory of Medical Molecular Diagnostics, Guangdong Medical University, Dongguan, China \\ ${ }^{3}$ Institute of Biochemistry \& Molecular Biology, Guangdong Medical University, Zhanjiang, China \\ ${ }^{4}$ Department of Clinical Laboratory, The Affiliated Hospital of Guangdong Medical University, Zhanjiang, China \\ ${ }^{5}$ Department of Cardiovascular Disease, The First People's Hospital of Foshan, Foshan, China \\ ${ }^{6}$ Department of Cardiovascular Disease, The Affiliated Hospital of Guangdong Medical University, Zhanjiang, China
}

Correspondence should be addressed to Xinguang Liu; xgliu64@126.com and Xing-dong Xiong; xiongxingdong@126.com

Received 13 September 2016; Revised 25 December 2016; Accepted 22 January 2017; Published 14 February 2017

Academic Editor: Michele Malaguarnera

Copyright (C) 2017 Shun Xu et al. This is an open access article distributed under the Creative Commons Attribution License, which permits unrestricted use, distribution, and reproduction in any medium, provided the original work is properly cited.

CXCL16 has been demonstrated to be involved in the development of atherosclerosis and myocardial infarction (MI). Nonetheless, the role of the CXCL16 polymorphisms on MI pathogenesis is far to be elucidated. We herein genotyped four tagSNPs in CXCL16 gene (rs2304973, rs1050998, rs3744700, and rs8123) in $275 \mathrm{MI}$ patients and 670 control subjects, aimed at probing into the impact of CXCL16 polymorphisms on individual susceptibility to MI. Multivariate logistic regression analysis showed that C allele $(\mathrm{OR}=1.31$, $95 \% \mathrm{CI}=1.03-1.66$, and $P=0.029)$ and $\mathrm{CC}$ genotype $(\mathrm{OR}=1.84,95 \% \mathrm{CI}=1.11-3.06$, and $P=0.018)$ of rs 1050998 were associated with increased MI risk; and C allele $(\mathrm{OR}=0.77,95 \% \mathrm{CI}=0.60-0.98$, and $P=0.036)$ of rs 8123 exhibited decreased MI risk, while the other two tagSNPs had no significant effect. Consistently, the haplotype rs2304973T-rs1050998C-rs3744700G-rs8123A containing the $\mathrm{C}$ allele of rs1050998 and A allele of rs8123 exhibited elevated MI risk $(\mathrm{OR}=1.41,95 \% \mathrm{CI}=1.02-1.96$, and $P=0.037)$. Further stratified analysis unveiled a more apparent association with MI risk among younger subjects ( $\leq 60$ years old). Taken together, our results provided the first evidence that CXCL16 polymorphisms significantly impacted MI risk in Chinese subjects.

\section{Introduction}

Myocardial infarction (MI), a main manifestation of coronary artery disease (CAD), poses increasing pressure on public health worldwide. Numerous environmental factors, such as obesity, hypercholesterolemia, alcohol intake, smoking, diabetes, and hypertension, have been established to contribute to the development of MI [1, 2]. Moreover, in addition to these modifiable factors, there is a growing body of studies having focused on the influence of genetic variants or polymorphisms within candidate genes in MI pathogenesis and thus yielding accumulating evidences that polymorphic variants in host genes exert crucial roles on the risk of MI $[3,4]$.

CXCL16, a newly discovered cytokine belonging to the CXC chemokine family, is expressed in both transmembrane and soluble forms [5]. As a transmembrane molecule, CXCL16 (also known as SR-PSOX) acts as a scavenger receptor for oxidized low-density lipoprotein (oxLDL) uptake, suggesting the involvement of CXCL16 in lipid metabolism [6]. While in a soluble form, CXCL16 has been found to interact with its receptor, CXCR6, and thus functions as an attractant and adhesion molecule for CXCR6-expressing T cells, which contribute to the development of atherosclerosis $[7,8]$. Mounting evidences have uncovered the close association of CXCL16 with the development of diverse human inflammatory diseases, including atherosclerosis [9], coronary artery disease [10], and MI [11]. Enhanced expression of both CXCL16 and CXCR6 has been observed in atherosclerotic lesions from humans as well as from apolipoprotein E(apoE-) deficient mice [12]. And the elevated expression level 
of CXCL16 was observed in MI patients as well [11]. Moreover, it has been reported that soluble CXCL16 in plasma could serve as a biomarker for acute coronary syndromes [13]. Thus it was reasonable to speculate that CXCL16 polymorphisms might probably exert an important role in MI pathogenesis.

Though the association between CXCL16 and MI pathogenesis has been fully studied, the effect of polymorphic variants in CXCL16 gene on the individual susceptibility to MI and its underlying molecular mechanisms are far to be elucidated. Thus, we herein conducted a case-control study to explore the association of the four tagSNPs in CXCL16 genes (rs2304973, rs1050998, rs3744700, and rs8123) with the risk of MI. Our data unraveled that the C allele of rs1055998 and the haplotype rs2304973T-rs1050998C-rs3744700G-rs8123A conferred an increased risk of $\mathrm{MI}$ in the Chinese Han population.

\section{Materials and Methods}

2.1. Study Subjects. A total of 945 Chinese Han subjects ( 275 MI patients and 670 control subjects) were included in our case-control study, who were consecutively recruited from the Affiliated Hospital of Guangdong Medical University (Zhanjiang, China) and the First People's Hospital of Foshan (Foshan, China) from March 2011 to December 2015. The diagnosis of MI was described previously [3]. 670 control subjects were recruited for regular physical examinations during the same period when MI patients were recruited. The 670 control subjects were judged to be free of MI by questionnaires, medical history, clinical examination, and electrocardiography. The individuals with a history of hematologic, renal, neoplastic, liver, or thyroid diseases were excluded.

All study subjects were genetically unrelated ethnic Han Chinese. Each subject was interviewed to collect information on demographic data and risk factors related to MI after obtaining the informed consent. The study was approved by the Medical Ethics Committee of the First People's Hospital of Foshan and the Affiliated Hospital of Guangdong Medical University.

2.2. Biochemical Parameters Analysis. An approximately $2 \mathrm{~mL}$ peripheral blood sample was drawn from each subject into tubes containing ethylenediaminetetraacetic acid (EDTA) after obtaining the informed consent. Immediately after collection, the blood sample was centrifuged at $2000 \times \mathrm{g}$ for $15 \mathrm{~min}$ and stored at $-80^{\circ} \mathrm{C}$. The levels of plasma total cholesterol (TC), triglyceride (TG), high density lipoprotein cholesterol (HDL-C), and low-density lipoprotein cholesterol (LDL-C) were measured enzymatically using a chemistry analyzer (Olympus, Japan). Glucose was analyzed by the glucose oxidase method with an Abbott V/P Analyzer (Abbott Laboratories, USA).

2.3. DNA Extraction. Genomic DNA was extracted from peripheral whole blood utilizing TIANamp blood DNA extraction kit (TianGen Biotech, China) according to the manufacturer's recommendations. All DNA samples were dissolved in water and stored at $-20^{\circ} \mathrm{C}$ until use.
2.4. TagSNP Selection and Genotyping. The Chinese Han population's SNP data of CXCL16 gene were downloaded from the HapMap database (http://www.hapmap.org). Then the SNP data of CXCL16 gene were analyzed using Haploview software version 4.2 [14] and obtained four tagSNPs, including rs2304973, rs1050998, rs3744700, and rs8123 (Figure 1(a)). A minor allele frequency (MAF) $>0.05$ and a linkage disequilibrium measure $\left(r^{2}\right)>0.8$ were prerequisites for tagSNPs selection $\left(r^{2}\right.$ values were shown in Figure 1(c)). These four tagSNPs would capture the information of the 8 known CXCL16 SNPs with a MAF > 0.05 (Figure 1(b)). Furthermore, the haplotypic blocks of the four tagSNPs were performed with the SHEsis platform [15].

The genotyping of the four tagSNPs was performed utilizing polymerase chain reaction-ligase detection reaction (PCR-LDR) method (Shanghai Biowing Applied Biotechnology Company), as described previously [16]. The sequences of primers and probes were listed in Table S1 in Supplementary Material available online at https://doi.org/10.1155/2017/ 9463272 .

2.5. Statistical Analysis. All the four tagSNPs of CXCL16 gene were tested for confirmation using Hardy-Weinberg expectations by a goodness-of-fit $\chi^{2}$ test among the control subjects. Quantitative variables were expressed as mean \pm standard deviation (SD), and qualitative variables were expressed as percentages. The differences of the demographic characteristics between the cases and controls were estimated by the $\chi^{2}$ test (for categorical variables) and Student's $t$-test (for continuous variables).

For the association analysis of individual tagSNP with MI risk, genotype frequencies were assessed by means of multivariate methods based on logistic regression analysis. And the odds ratios (ORs) and 95\% confidence intervals (CIs) for the effect of SNPs on MI risk were adjusted by age, sex, smoking, drinking, hypertension, diabetes, and hyperlipidemia. The statistical analyses were performed using the SPSS software (version 19). The haplotype analysis on the polymorphisms was done using SHEsis software freely available at (http://analysis.bio-x.cn/myAnalysis.php). $P$ value of less than 0.05 was used as the criterion of statistical significance.

\section{Results}

3.1. Characteristics of the Study Population. The characteristics of the enrolled subjects in the study (275 MI cases and 670 control subjects) were listed in Table 1. In comparison with control subjects, the MI patients exhibited higher proportion of male gender, smokers, and alcohol consumers $(P<0.001$, $P<0.001$, and $P<0.001$, resp.), more prevalence of hypertension, hyperlipidemia and diabetes $(P<0.001, P<0.001$, and $P<0.001$, resp.), and higher levels of fasting plasma glucose (FPG), triglycerides (TG), and LDL-C $(P<0.001, P<$ 0.001 , and $P<0.001$, resp.) but lower HDL-C $(P<0.001)$, while no statistically significant difference between cases and controls was observed in terms of age $(P=0.483)$ and TC levels $(P=0.175)$. In all, these data further demonstrated that male gender, smoking, alcohol intake, hypertension, 


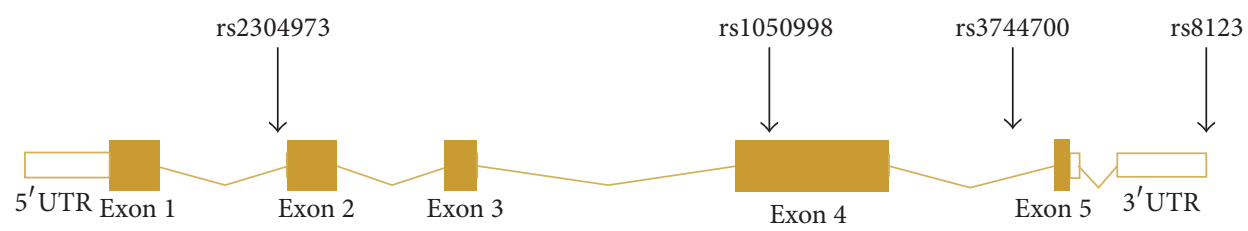

(a)

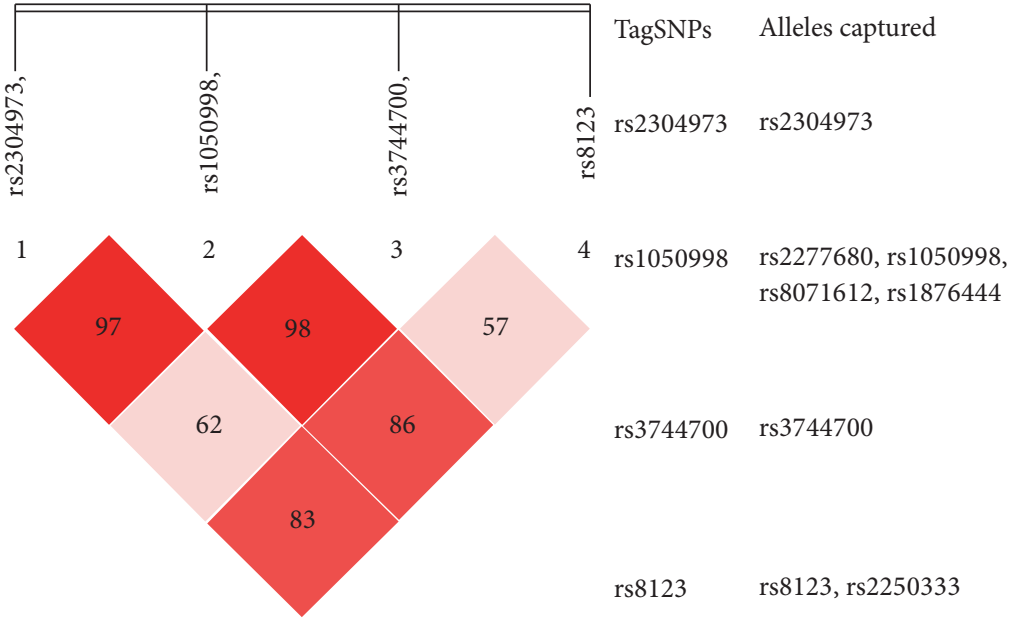

(b)
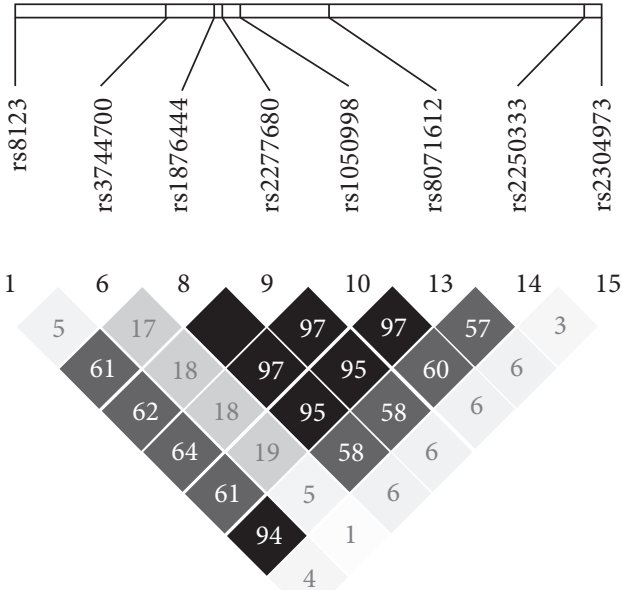

(c)

FIGURE 1: Schematic of CXCL16 gene structure and pairwise LD between the four tagSNPs. (a) Schematic of the CXCL16 gene structure and the location of the four tagSNPs (rs2304973, rs1050998, rs3744700, and rs8123) within CXCL16 gene. (b) $D^{\prime}$ values are plotted as a graph to show linkage disequilibrium among the four tagSNPs. Details of the selected tagSNPs and respective SNPs captured by the four tagSNPs are also indicated. (c) The $r^{2}$ values are plotted as a graph to show the linkage disequilibrium measure among the eight SNPs (rs8123, rs3744700, rs1876444, rs2277680, rs1050998, rs8071612, rs2250333, and rs2304973) captured by these four tagSNPs within CXCL16 gene.

hyperlipidemia, and diabetes mellitus were the critical risk factors for MI development in Chinese population.

\subsection{Multivariate Associations of Four tagSNPs with the Risk} of MI. Four tagSNPs (rs2304973, rs1050998, rs3744700, and rs8123) in CXCL16 gene were genotyped in $275 \mathrm{MI}$ patients and 670 control subjects. The primary information for these four polymorphisms was listed in Table 2. Minor allele frequency (MAF) of all four tagSNPs in the control subjects was similar to MAF for Chinese in HapMap database (Table 2). All the genotype frequency distributions of the four tagSNPs in the controls followed Hardy-Weinberg equilibrium proportions (all $P$ values $\geq 0.10$, Table 2 ).

The allele and genotype distributions of the four tagSNPs in the cases and controls were presented in Table 3 . The allelic association analysis uncovered that the C allele of rs1050998 was associated with an evidently enhanced risk of MI (OR $=1.31,95 \% \mathrm{CI}=1.03-1.66$, and $P=0.029$, Table 3$)$. In addition, compared to TT genotype, the CC homozygote (OR $=1.84,95 \% \mathrm{CI}=1.11-3.06$, and $P=0.018$, Table 3 ) or CT heterozygote $(\mathrm{OR}=1.67,95 \% \mathrm{CI}=1.03-2.70$, and $P=0.037$, Table 3) exhibited an increased risk of MI as well. Moreover, the $\mathrm{C}$ allele of rs8123 conferred a diminished risk of $\mathrm{MI}$ compared to A allele $(\mathrm{OR}=0.77,95 \% \mathrm{CI}=0.60-0.98$, and $P=$ 0.036 , Table 3). Consistently, the CC as well as combined AC + CC genotypes showed borderline significantly decreased risk for MI (Table 3, OR $=0.58,95 \% \mathrm{CI}=0.34-1.01$, and $P=0.054$ and $\mathrm{OR}=0.73,95 \% \mathrm{CI}=0.52-1.02$, and $P=0.065$, resp.), in comparison with the GG genotype. In all, our data indicated that CXCL16 tagSNPs were closely associated with MI risk in the Chinese Han population. And individuals carrying C allele of rs1050998 exhibited significantly increased MI susceptibility, while the $\mathrm{C}$ allele of $\mathrm{rs} 8123$ potentially provided a protective effect against MI risk. However, no significant association between rs2304973 and rs3744700 and MI risk was observed (Table 3).

3.3. Stratification Analyses of CXCL16 rs1050998 and rs8123 Polymorphism and Risk of MI. We further evaluated the alleles or genotypes of CXCL16 rs1050998 and rs8123 and MI susceptibility after stratifying the subjects by age, sex, status of smoking, or drinking. Stratification analyses by age ( $\leq 60$ or $>60$ years old) unveiled that the increased MI risk of individuals carrying $\mathrm{C}$ allele $(\mathrm{OR}=1.59,95 \% \mathrm{CI}=1.08-$ 2.35 , and $P=0.020$, Table 4 ) or CC genotype ( $\mathrm{OR}=2.49$, 95\% CI $=1.11-5.59$, and $P=0.027$, Table 4 ) of rs1050998 was more notable among younger subjects ( $\leq 60$ years old) whereas no significant association was observed from the group older than 60 years old (Table 4 ). And the protective effect of $\mathrm{C}$ allele $(\mathrm{OR}=0.60,95 \% \mathrm{CI}=0.40-0.89$, and $P=0.012$, Table 4$)$ or CC genotype $(\mathrm{OR}=0.38,95 \% \mathrm{CI}$ 
TABLE 1: The characteristics of MI cases and controls.

\begin{tabular}{|c|c|c|c|}
\hline Variable & Controls $(n=670)$ & Cases $(n=275)$ & $P^{\mathrm{a}}$ \\
\hline Age (years) & $61.48 \pm 12.31$ & $62.10 \pm 12.00$ & 0.483 \\
\hline Sex (male) & $387(57.8 \%)$ & $213(77.5 \%)$ & $<0.001^{\mathrm{b}}$ \\
\hline Smoking & $174(26.0 \%)$ & $163(59.3 \%)$ & $<\mathbf{0 . 0 0 1}$ \\
\hline Drinking & $95(14.2 \%)$ & $73(26.5 \%)$ & $<0.001$ \\
\hline Hypertension & $239(35.7 \%)$ & $171(62.2 \%)$ & $<0.001$ \\
\hline Diabetes & $107(16.0 \%)$ & $129(46.9 \%)$ & $<0.001$ \\
\hline Hyperlipidemia & $253(37.8 \%)$ & $195(70.9 \%)$ & $<0.001$ \\
\hline Systolic BP (mmHg) & $132.56 \pm 18.91$ & $140.53 \pm 18.77$ & $<0.001$ \\
\hline Diastolic BP (mmHg) & $72.97 \pm 10.47$ & $75.93 \pm 10.87$ & $<0.001$ \\
\hline $\mathrm{FPG}(\mathrm{mM})$ & $5.80 \pm 1.88$ & $6.61 \pm 1.70$ & $<0.001$ \\
\hline Triglycerides (mM) & $1.49 \pm 0.81$ & $2.07 \pm 0.96$ & $<0.001$ \\
\hline Total cholesterol (mM) & $4.62 \pm 1.15$ & $4.73 \pm 1.19$ & 0.175 \\
\hline LDL cholesterol (mM) & $2.64 \pm 0.90$ & $3.04 \pm 0.97$ & $<0.001$ \\
\hline HDL cholesterol (mM) & $1.37 \pm 0.66$ & $1.20 \pm 0.40$ & $<0.001$ \\
\hline
\end{tabular}

${ }^{\mathrm{a}}$ Two-sided chi-square test or independent-samples $t$-test.

${ }^{\mathrm{b}} P$ values under 0.05 were indicated in bold font.

TABLE 2: Primary information for rs2304973, rs1050998, rs3744700, and rs8123 SNPs.

\begin{tabular}{|c|c|c|c|c|}
\hline Genotyped SNPs & rs2304973 & rs1050998, & rs3744700 & rs8123 \\
\hline Chr Pos (Genome Build 107.0) & 4738927 & 4735442 & 4734715 & 4733270 \\
\hline Pos in Cxcl16 gene & Intron 1 & Extron 4 & Intron 4 & nearGene-3 \\
\hline $\mathrm{MAF}^{\mathrm{a}}$ for Chinese (CHB) in HapMap & 0.089 & 0.451 & 0.134 & 0.291 \\
\hline MAF in our controls $(n=670)$ & 0.089 & 0.437 & 0.110 & 0.369 \\
\hline$P$ value for $\mathrm{HWE}^{\mathrm{b}}$ test in our controls & 0.540 & 0.281 & 0.444 & 0.370 \\
\hline
\end{tabular}

${ }^{a} \mathrm{MAF}$ : minor allele frequency.

${ }^{\mathrm{b}}$ HWE: Hardy-Weinberg equilibrium.

$=0.16-0.93$, and $P=0.031$, Table 4$)$ of rs8123 was more evident among younger subjects ( $\leq 60$ years old) as well. No more significant association between CXCL16 rs1050998 and rs8123 polymorphism and risk of MI was observed among subgroups by sex, status of smoking, or drinking (data not shown).

3.4. Association between the Haplotypes of CXCL16 tagSNPS with the Risk of MI. As shown in Figure 1(b), all the four tagSNPs were located in one haplotypic block. We thus further compared the haplotype frequencies of the four tagSNPs between MI group and controls. Four common haplotypes (frequency $>3 \%$ ) derived from the four tagSNPs accounted for approximately $96 \%$ of the haplotype variations (Table 5). Consistently, among the four common haplotypes, the haplotype containing C allele of rs1050998 and A allele of rs8123 (rs2304973T-rs1050998C-rs3744700G-rs8123A) was found to be associated with an increased risk for $\mathrm{MI}(\mathrm{OR}=1.41,95 \%$ $\mathrm{CI}=1.02-1.96$, and $P=0.037$, Table 5); and the haplotype containing $\mathrm{T}$ allele of rs1050998 and $\mathrm{C}$ allele of rs8123 (rs2304973C-rs1050998T- rs3744700G-rs8123C) exhibited a reduced $\mathrm{MI}$ risk $(\mathrm{OR}=0.77,95 \% \mathrm{CI}=0.62-0.96$, and $P=$ 0.022 , Table 5).

\section{Discussion}

Previous studies have established the close association between CXCL16 and the pathogenesis of atherosclerosis and MI. Nonetheless, the impact of tagSNPs in CXCL16 gene on MI risk is still largely unknown. In this study, we performed a genetic association analysis on the four tagSNPs (rs2304973, rs1050998, rs3744700, and rs8123) within CXCL16 gene and unraveled that the $\mathrm{C}$ allele of rs1050998 and the A allele of rs8123 and the haplotype rs2304973T-rs1050998Crs3744700G-rs8123A containing C allele of rs1050998 and the A allele of rs8123 conferred enhanced risk of MI in the Chinese Han population. Moreover, the association between CXCL16 polymorphisms and MI risk was more remarkable among younger subjects ( $\leq 60$ years old). These data indicated that the C allele of rs1050998 and the A allele of rs8123 might significantly enhance the risk of MI in the Chinese Han population.

The association of polymorphisms of the CXCL16 gene locus with various inflammatory diseases has been widely studied [17]. However, the effect of CXCL16 tagSNPs on MI risk is still unknown. Zivković et al. have reported that the rs1050998 (I142T) polymorphisms were significantly associated with the occurrence of Carotid Atherosclerosis (CA) plaque $(\mathrm{OR}=1.27, P=0.03)$ [9]. Our data indicated that 
TABLE 3: Multivariate associations of the four tagSNPs in CXCL16 gene with the risk of MI.

\begin{tabular}{|c|c|c|c|c|}
\hline Type & $\begin{array}{c}\text { Controls }(n=670) \\
\text { Number }(\%)\end{array}$ & $\begin{array}{c}\text { Cases }(n=275) \\
\text { Number }(\%)\end{array}$ & OR $(95 \% \mathrm{CI})^{\mathrm{a}}$ & $P$ value $^{\mathrm{a}}$ \\
\hline \multicolumn{5}{|l|}{ rs2304973 } \\
\hline C & $1221(91.1)$ & $485(88.2)$ & 1.00 & - \\
\hline $\mathrm{T}$ & $119(8.9)$ & $65(11.8)$ & $1.46(1.00-2.12)$ & 0.050 \\
\hline $\mathrm{CC}$ & $555(82.8)$ & $216(78.5)$ & 1.00 & - \\
\hline $\mathrm{CT}$ & $111(16.6)$ & $53(19.3)$ & $1.31(0.85-2.00)$ & 0.220 \\
\hline $\mathrm{TT}$ & $4(0.6)$ & $6(2.2)$ & $4.16(0.96-18.03)$ & 0.057 \\
\hline $\mathrm{CC}$ & $555(82.8)$ & $216(78.5)$ & 1.00 & - \\
\hline $\mathrm{CT}+\mathrm{TT}$ & $115(17.2)$ & $59(21.5)$ & $1.44(0.96-2.17)$ & 0.080 \\
\hline \multicolumn{5}{|l|}{ rs1050998 } \\
\hline $\mathrm{T}$ & $586(43.7)$ & $215(39.1)$ & 1.00 & - \\
\hline $\mathrm{C}$ & $754(56.3)$ & $335(60.9)$ & $1.31(1.03-1.66)$ & 0.029 \\
\hline TT & $135(20.1)$ & $38(13.8)$ & 1.00 & - \\
\hline $\mathrm{CT}$ & $316(47.2)$ & $139(50.5)$ & $1.67(1.03-2.70)$ & 0.037 \\
\hline $\mathrm{CC}$ & $219(32.7)$ & $98(35.6)$ & $1.84(1.11-3.06)$ & $0.018^{\mathrm{b}}$ \\
\hline $\mathrm{TT}$ & $135(20.1)$ & $38(13.8)$ & 1.00 & - \\
\hline $\mathrm{CT}+\mathrm{CC}$ & $535(79.9)$ & $237(86.2)$ & $1.74(1.10-2.75)$ & 0.018 \\
\hline \multicolumn{5}{|l|}{ rs3744700 } \\
\hline G & $1193(89.0)$ & $487(88.5)$ & 1.0 & - \\
\hline $\mathrm{T}$ & $147(11.0)$ & $63(11.5)$ & $1.05(0.72-1.51)$ & 0.811 \\
\hline GG & $533(79.6)$ & $216(78.5)$ & 1.0 & - \\
\hline GT & $127(19.0)$ & $55(19.3)$ & $1.02(0.68-1.56)$ & 0.905 \\
\hline $\mathrm{TT}$ & $10(1.5)$ & $4(1.5)$ & $0.80(0.19-3.46)$ & 0.767 \\
\hline GG & $533(79.6)$ & $216(78.5)$ & 1.0 & - \\
\hline $\mathrm{GT}+\mathrm{TT}$ & $137(20.4)$ & $59(21.5)$ & $1.04(0.69-1.56)$ & 0.857 \\
\hline \multicolumn{5}{|l|}{$r s 8123$} \\
\hline A & $845(63.1)$ & $377(68.5)$ & 1.00 & - \\
\hline $\mathrm{C}$ & 495 (36.9) & $173(31.5)$ & $0.77(0.60-0.98)$ & 0.036 \\
\hline AA & $269(40.1)$ & $130(47.3)$ & 1.00 & - \\
\hline $\mathrm{AC}$ & $307(45.8)$ & $117(42.5)$ & $0.78(0.55-1.11)$ & 0.163 \\
\hline $\mathrm{CC}$ & $94(14.0)$ & $28(10.2)$ & $0.58(0.34-1.01)$ & 0.054 \\
\hline AA & $269(40.1)$ & $130(47.3)$ & 1.00 & - \\
\hline $\mathrm{AC}+\mathrm{CC}$ & $401(59.9)$ & $145(52.7)$ & $0.73(0.52-1.02)$ & 0.065 \\
\hline
\end{tabular}

${ }^{a}$ Adjusted for age, sex, smoking, drinking, hypertension, diabetes, and hyperlipidemia.

${ }^{\mathrm{b}} P$ values under 0.05 were indicated in bold font.

individuals carrying C allele of rs1050998 exhibited enhanced MI risk, which was consistent with the previously published literature [9]. Another study suggested that the rs2304973 showed no significant difference between CAD patients and control subjects [18], which was compatible with our results that there is no evident association of rs2304973 with the risk of MI. In addition, the rs3744700 polymorphism has been reported to be closely related to the development of CAD $(\mathrm{OR}=1.77, P<0.001)[18,19]$; however, there is no significant association between rs3744700 and MI risk in our case-control study, which might be due to the difference between CAD and MI.

As shown that both rs1050998 and rs8123 tagSNPs capture other closely linked SNPs (high LD) within or near the CXCL16 gene locus (Figure 1), thus the association of rs1050998 and rs8123 polymorphisms with MI risk might be direct due to their causative effect or because of the other functional polymorphisms captured by them. The rs 2277680 polymorphism captured by rs1050998 has been unveiled to have a marginal association with the risk of Crohn's disease (CD) in patients $(P=0.0482, \mathrm{OR}=1.4310)$ [20] but exhibited no significant association with CAD risk [18]. Similarly, no significant difference was observed for the distribution of the rs2250333 polymorphism captured by rs 8123 between CAD patients and control subjects as well [18]. We noticed that the CXCL16 rs1050998 (T/C) polymorphism caused the Tto- $C$ change, which resulted in the missense mutation of I (Ile) $142 \mathrm{~T}$ (Thr). One single amino acid mutation might extensively impact the structure, stability, and activity of the protein [21-23], especially when the amino acid changed between nonpolar amino acid (Ile) and polar amino acid (Thr) [24]. Thus, it is reasonable to speculate that the 
TABLE 4: Multivariate associations of the rs1050998 and rs8123 in CXCL16 gene with the risk of MI by further stratification for age.

\begin{tabular}{|c|c|c|c|c|}
\hline \multirow{2}{*}{ Genotype } & \multicolumn{2}{|c|}{ Age $\leq 60$} & \multicolumn{2}{|c|}{ Age $>60$} \\
\hline & OR $(95 \% \mathrm{CI})^{\mathrm{a}}$ & $P$ value $^{\mathrm{a}}$ & OR $(95 \% \mathrm{CI})^{\mathrm{b}}$ & $P$ value \\
\hline \multicolumn{5}{|l|}{ rs1050998 } \\
\hline $\mathrm{T}$ & 1.00 & - & 1.00 & - \\
\hline $\mathrm{C}$ & $1.59(1.08-2.35)$ & $0.020^{b}$ & $1.19(0.87-1.61)$ & 0.273 \\
\hline $\mathrm{TT}$ & 1.00 & - & 1.00 & - \\
\hline $\mathrm{CT}$ & $1.50(0.70-3.24)$ & 0.300 & $1.78(0.94-3.36)$ & 0.075 \\
\hline $\mathrm{CC}$ & $2.49(1.11-5.59)$ & 0.027 & $1.60(0.83-3.08)$ & 0.161 \\
\hline \multicolumn{5}{|l|}{ rs8123 } \\
\hline A & 1.00 & - & 1.00 & - \\
\hline $\mathrm{C}$ & $0.60(0.40-0.89)$ & 0.012 & $0.87(0.63-1.19)$ & 0.377 \\
\hline AA & 1.00 & - & 1.00 & - \\
\hline $\mathrm{AC}$ & $0.56(0.32-0.98)$ & 0.041 & $0.91(0.57-1.46)$ & 0.701 \\
\hline $\mathrm{CC}$ & $0.38(0.16-0.93)$ & 0.031 & $0.72(0.36-1.45)$ & 0.359 \\
\hline
\end{tabular}

${ }^{a}$ Adjusted for sex, smoking, drinking, hypertension, diabetes, and hyperlipidemia.

${ }^{\mathrm{b}} P$ values under 0.05 were indicated in bold font.

TABLE 5: Association between haplotypes of the four tagSNPs in CXCL16 gene with the risk of MI.

\begin{tabular}{|c|c|c|c|c|}
\hline \multirow{2}{*}{ Haplotype $^{a}$} & Controls $(n=670)$ & Cases $(n=275)$ & \multirow{2}{*}{ OR (95\% CI) } & \multirow{2}{*}{$P$} \\
\hline & Number (\%) & Number (\%) & & \\
\hline C C G A & $604.21(45.1)$ & $257.74(46.9)$ & $1.08(0.88-1.32)$ & 0.482 \\
\hline C T G C & $438.97(32.8)$ & $150.95(27.4)$ & $0.77(0.62-0.96)$ & $0.022^{\mathrm{b}}$ \\
\hline C T T A & $126.76(9.5)$ & $54.96(10.0)$ & $1.06(0.76-1.48)$ & 0.772 \\
\hline T C G A & $112.85(8.3)$ & $63.25(11.5)$ & $1.41(1.02-1.96)$ & 0.037 \\
\hline
\end{tabular}

${ }^{a}$ The allelic sequence in the haplotypes is in the following order: rs2304973, rs1050998, rs3744700, and rs8123.

${ }^{\mathrm{b}} P$ values under 0.05 were indicated in bold font.

rs1050998 polymorphism might exert a direct causative effect on the MI risk.

The stratified analyses of the association of rs1050998 and rs8123 polymorphisms with MI risk revealed that the increased risk of CXCL16 rs150998 and rs8123 in MI was more remarkable among younger subjects ( $\leq 60$ years old), while no significant association was observed in the older group (>60 years old) (Table 4 ). This phenomenon was similar to our previous study, which uncovered that the enhanced risk conferred by LRP6 rs rs2302685 in MI was more evident among younger subjects ( $\leq 60$ years old) as well [3]. The potential explanation to this phenomenon was that the dominant cause of MI pathogenesis in older subjects is more likely due to the aging effects rather than direct genetic effects.

There are several limitations in this case-control study that need to be addressed. Initially, the possibility that the subjects (275 MI patients and 670 control subjects) enrolled from hospitals may not represent the general population could not be excluded. Nevertheless, the distributions of the selected tagSNPs in the controls were in Hardy-Weinberg equilibrium. Second, the relatively small sample size limited the statistical power of this study, especially for the case subjects. Finally, further investigations in different population and with larger sample size contribute to verifying the general validity of our findings. However, the results drawn from our case-control study provided novel insights and fascinating information for further studies in this area.

\section{Conclusions}

Taken together, our case-control study firstly provides the evidences that the CXCL16 polymorphisms significantly impacted the risk of $\mathrm{MI}$ in the Chinese Han population, and the association between CXCL16 polymorphisms and MI risk was more evident among younger subjects.

\section{Abbreviations}

CXCL16: CXC motif chemokine ligand 16

SNP: $\quad$ Single nucleotide polymorphism

MI: $\quad$ Myocardial infarction

CAD: $\quad$ Coronary artery disease

PCR-LDR: Polymerase chain reaction-ligase detection reaction

OR: Odds ratio

CI: Confidence interval

LDL-C: Low-density lipoprotein cholesterol

TC: $\quad$ Total cholesterol

TG: $\quad$ Triglyceride

HDL-C: High density lipoprotein cholesterol. 


\section{Competing Interests}

The authors declare no conflict of interests.

\section{Acknowledgments}

The authors thank the First People's Hospital of Foshan and the Affiliated Hospital of Guangdong Medical University, Guangdong Province, China, for their kind assistance in collecting the samples and data. This work was supported by grants from the National Natural Science Foundation of China (81370456, 31600976), the Natural Science Foundation of Guangdong Province (2014A030311015, 2014KZDXM041, 2014A030310027, and 2016A030313684), Yangfan Training Program of Guangdong Province (4YF16006G), the Science and Technology Planning Project of Dongguan City (2015108101015, 2013108101057), and the Medical Scientific Research Foundation of Guangdong Province (A2015288).

\section{References}

[1] X.-H. Zhang, Z. L. Lu, and L. Liu, "Coronary heart disease in China," Heart, vol. 94, no. 9, pp. 1126-1131, 2008.

[2] R. Ramaraj, "Risk factors for myocardial infarction in women and men," European Heart Journal, vol. 30, no. 8, pp. 1012-1013, 2009.

[3] S. Xu, J. Cheng, Y.-N. Chen et al., "The LRP6 rs2302685 polymorphism is associated with increased risk of myocardial infarction," Lipids in Health and Disease, vol. 13, no. 1, article 94, 2014.

[4] Z. Juan, Z. Wei-Guo, S. Heng-Liang, and W. Da-Guo, "Association of matrix metalloproteinase $9 \mathrm{C}-1562 \mathrm{~T}$ polymorphism with genetic susceptibility to myocardial infarction: a meta-analysis," Current Therapeutic Research - Clinical and Experimental, vol. 77, pp. 40-45, 2015.

[5] A. E. Norlander, M. A. Saleh, and M. S. Madhur, "CXCL16: a chemokine-causing chronic kidney disease," Hypertension, vol. 62, no. 6, pp. 1008-1010, 2013.

[6] A. M. Jansson, P. Aukrust, T. Ueland et al., "Soluble CXCL16 predicts long-term mortality in acute coronary syndromes," Circulation, vol. 119, no. 25, pp. 3181-3188, 2009.

[7] P. Seizer, K. Stellos, G. Selhorst et al., "CXCL16 is a novel scavenger receptor on platelets and is associated with acute coronary syndrome," Thrombosis and Haemostasis, vol. 105, no. 6, pp. 1112-1114, 2011.

[8] R. Yamauchi, M. Tanaka, N. Kume et al., "Upregulation of SRPSOX/CXCL16 and recruitment of $\mathrm{CD}^{+} \mathrm{T}$ cells in cardiac valves during inflammatory valvular heart disease," Arteriosclerosis, Thrombosis, and Vascular Biology, vol. 24, no. 2, pp. 282287, 2004.

[9] M. Zivković, T. Djurić, L. Stojković et al., "CXCL16 haplotypes in patients with human carotid atherosclerosis: preliminary results," Journal of Atherosclerosis and Thrombosis, vol. 22, no. 1, pp. 10-20, 2015.

[10] F. Zhou, J. Wang, K. Wang et al., "Serum CXCL16 as a novel biomarker of coronary artery disease in type 2 diabetes mellitus: a pilot study," Annals of Clinical and Laboratory Science, vol. 46, no. 2, pp. 184-189, 2016.

[11] L. E. Laugsand, B. O. Åsvold, L. J. Vatten et al., "Soluble CXCL16 and risk of myocardial infarction: The HUNT Study in Norway," Atherosclerosis, vol. 244, pp. 188-194, 2016.
[12] D. M. Wuttge, X. Zhou, Y. Sheikine et al., "CXCL16/SR-PSOX is an interferon- $\gamma$-regulated chemokine and scavenger receptor expressed in atherosclerotic lesions," Arteriosclerosis, Thrombosis, and Vascular Biology, vol. 24, no. 4, pp. 750-755, 2004.

[13] M. Lehrke, S. C. Millington, M. Lefterova et al., "CXCL16 is a marker of inflammation, atherosclerosis, and acute coronary syndromes in humans," Journal of the American College of Cardiology, vol. 49, no. 4, pp. 442-449, 2007.

[14] J. C. Barrett, B. Fry, J. Maller, and M. J. Daly, "Haploview: analysis and visualization of LD and haplotype maps," Bioinformatics, vol. 21, no. 2, pp. 263-265, 2005.

[15] Y. Y. Shi and L. He, "SHEsis, a powerful software platform for analyses of linkage disequilibrium, haplotype construction, and genetic association at polymorphism loci," Cell Research, vol. 15, no. 2, pp. 97-98, 2005.

[16] J. Cheng, M. Cho, J.-M. Cen et al., "A TagSNP in SIRT1 gene confers susceptibility to myocardial infarction in a Chinese Han population," PLoS ONE, vol. 10, no. 2, Article ID e0115339, 2015.

[17] J. Seiderer, J. Dambacher, D. Leistner et al., "Genotypephenotype analysis of the CXCL16 p.Ala181Val polymorphism in inflammatory bowel disease," Clinical Immunology, vol. 127, no. 1, pp. 49-55, 2008.

[18] M. Huang, Y. Han, X. Zhang et al., "An intron polymorphism in the CXCL16 gene is associated with increased risk of coronary artery disease in Chinese Han population: A lArge Angiography-based Study," Atherosclerosis, vol. 210, no. 1, pp. 160-165, 2010.

[19] J. Tian, S. Hu, F. Wang, X. Yang, Y. Li, and C. Huang, "PPARG, AGTR1, CXCL16 and LGALS2 polymorphisms are correlated with the risk for coronary heart disease," International Journal of Clinical and Experimental Pathology, vol. 8, no. 3, pp. 3138-3143, 2015.

[20] K. H. Chua, J. G. Ng, C. C. Ng, I. Hilmi, K. L. Goh, and B. P. Kee, "Association of NOD1, CXCL16, STAT6 and TLR4 gene polymorphisms with Malaysian patients with Crohn's disease," PeerJ, vol. 4, no. 3, article 1843, 2016.

[21] M. Tatsuguchi, M. Furutani, J.-I. Hinagata et al., "Oxidized LDL receptor gene (OLR1) is associated with the risk of myocardial infarction," Biochemical and Biophysical Research Communications, vol. 303, no. 1, pp. 247-250, 2003.

[22] Q. Zhang, Y. Du, J. Zhang et al., "Functional impact of 14 single nucleotide polymorphisms causing missense mutations of human $\alpha 7$ nicotinic receptor," PLoS ONE, vol. 10, no. 9, Article ID e0137588, 2015.

[23] M. Katoh, "Cancer genomics and genetics of FGFR2 (Review)," International Journal of Oncology, vol. 33, no. 2, pp. 233-237, 2008.

[24] J. Rebehmed, F. Quintus, J.-P. Mornon, and I. Callebaut, “The respective roles of polar/nonpolar binary patterns and amino acid composition in protein regular secondary structures explored exhaustively using hydrophobic cluster analysis," Proteins: Structure, Function and Bioinformatics, vol. 84, no. 5, pp. 624638, 2016. 


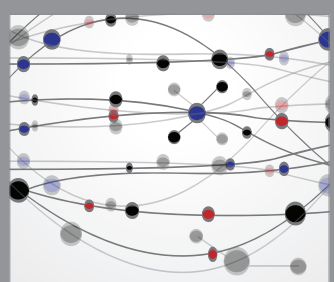

The Scientific World Journal
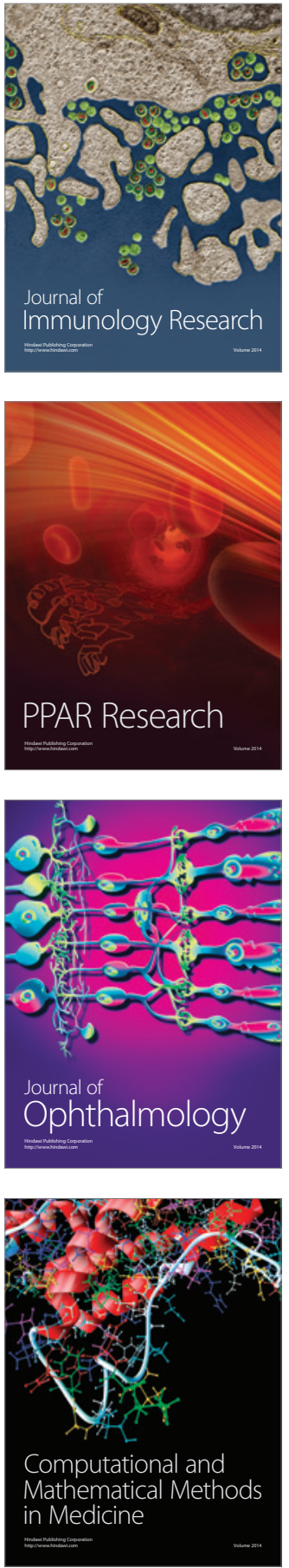

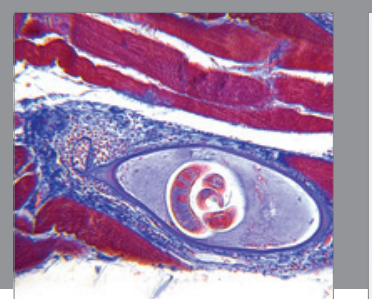

Gastroenterology Research and Practice
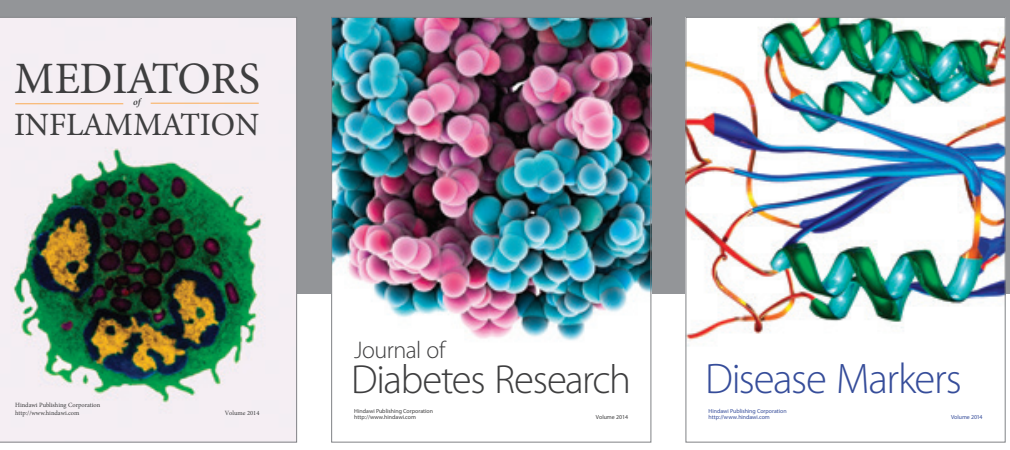

Disease Markers

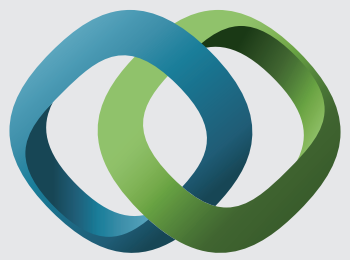

\section{Hindawi}

Submit your manuscripts at

https://www.hindawi.com
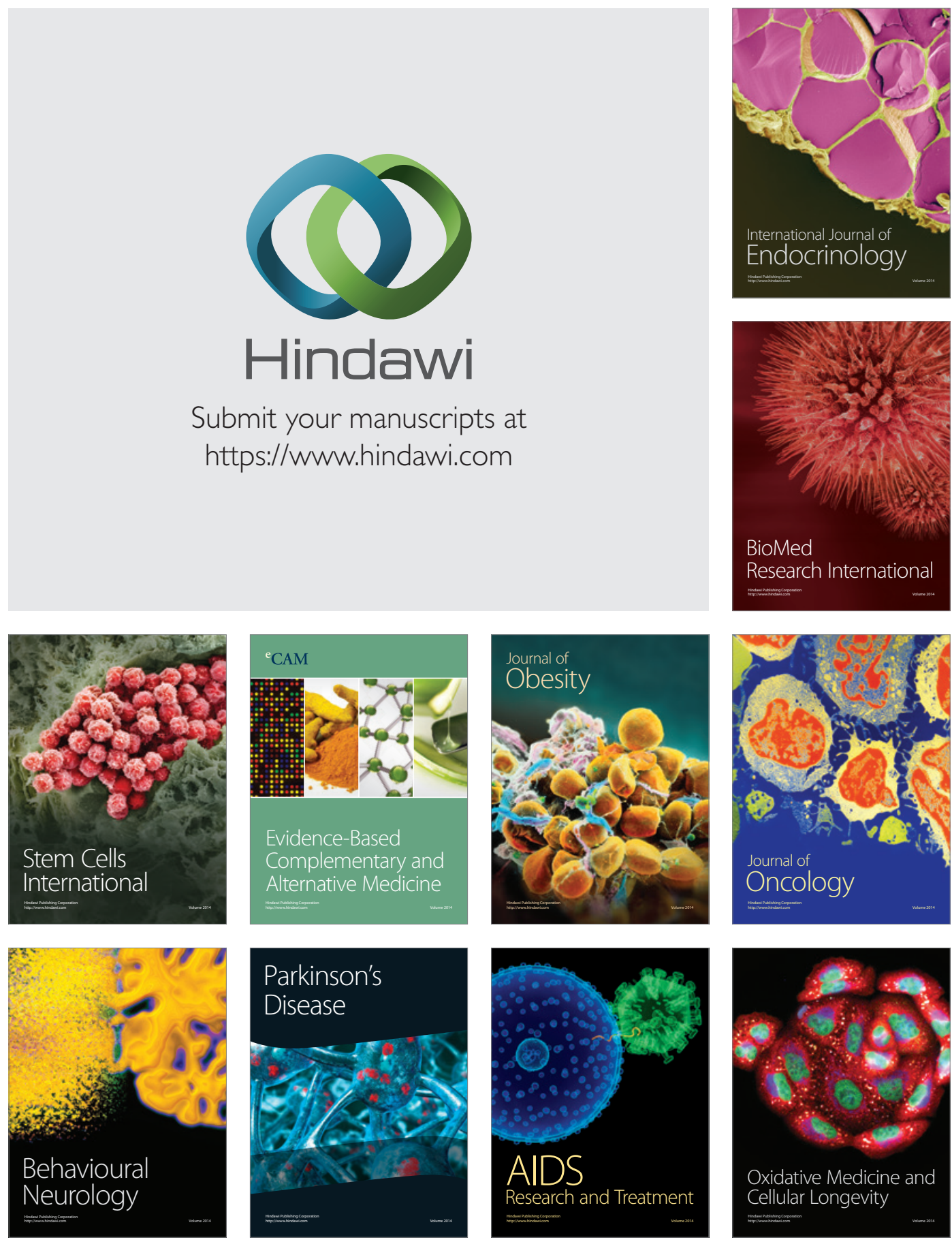